\title{
The Utilization of Robotic Pets in Dementia Care
}

\author{
Sandra Petersen ${ }^{\mathrm{a}, *}$, Susan Houston ${ }^{\mathrm{b}}$, Huanying Qin ${ }^{\mathrm{c}}$, Corey Tague $^{\mathrm{d}}$ and Jill Studley \\ ${ }^{\mathrm{a}}$ The University of Texas at Tyler, College of Nursing and Health Sciences, Tyler, TX, USA \\ ${ }^{\mathrm{b}}$ Baylor Scott and White Health Care System Office of the CNO, Plano, TX, USA \\ ${ }^{\mathrm{c}}$ Baylor Scott and White Health Care System, Dallas, TX, USA \\ ${ }^{\mathrm{d}}$ ACH Health Services, Hurst, TX, USA \\ ${ }^{\mathrm{e}}$ Baylor Elder HouseCalls, Dallas, TX, USA
}

Accepted 18 August 2016

\begin{abstract}
.
Background: Behavioral problems may affect individuals with dementia, increasing the cost and burden of care. Pet therapy has been known to be emotionally beneficial for many years. Robotic pets have been shown to have similar positive effects without the negative aspects of traditional pets. Robotic pet therapy offers an alternative to traditional pet therapy.

Objective: The study rigorously assesses the effectiveness of the PARO robotic pet, an FDA approved biofeedback device, in treating dementia-related symptoms.

Methods: A randomized block design with repeated measurements guided the study. Before and after measures included reliable, valid tools such as: RAID, CSDD, GDS, pulse rate, pulse oximetry, and GSR. Participants interacted with the PARO robotic pet, and the control group received standard activity programs. Five urban secure dementia units comprised the setting. Results: 61 patients, with 77\% females, average 83.4 years in age, were randomized into control and treatment groups. Compared to the control group, RAID, CSDD, GSR, and pulse oximetry were increased in the treatment group, while pulse rate, pain medication, and psychoactive medication use were decreased. The changes in GSR, pulse oximetry, and pulse rate over time were plotted for both groups. The difference between groups was consistent throughout the 12-week study for pulse oximetry and pulse rate, while GSR had several weeks when changes were similar between groups.

Conclusions: Treatment with the PARO robot decreased stress and anxiety in the treatment group and resulted in reductions in the use of psychoactive medications and pain medications in elderly clients with dementia.
\end{abstract}

Keywords: Animal assisted therapy, biofeedback, dementia, psychology, robotics

\section{INTRODUCTION}

According to Alzheimer's Disease International, the worldwide costs of dementia (\$604 billion in US dollars) amounted to more than $1 \%$ of the global gross domestic product in 2010. The worldwide cost is projected to reach $\$ 1$ trillion in 2018. Currently, over 46 million people are living with dementia. By 2050, this number will have risen to 131.5 million. Furthermore,

${ }^{*}$ Correspondence to: Sandra Petersen, DNP, RN, GNP-BC, FNP-BC, FAANP, Professor and Director, Doctor of Nursing Practice Program at UT Tyler, College of Nursing \& Health Sciences, 3900 University Blvd., Tyler, TX 75799, USA. Tel.: +1 214213 4726; Fax: +1 866672 8204; E-mail: spetersen@uttyler.edu. dementia patients have increased healthcare utilization rates compared with patients with other major diseases. Previous research shows that informal costs make up a substantial part of the total annual costs of dementia. In the United States alone, the care provided by informal caregivers to people with dementia was valued at more than $\$ 202$ billion in 2010 [1].

Behavioral and psychological problems affect most individuals with dementia at some point during the progression of the disorder, adding to the cost and burden of caring for them. For example, as many as $83 \%$ of individuals with dementia suffer with depression [2]; and as many as $77 \%$ suffer from anxiety [3]. These problems may ultimately result in 
long-term hospitalization, increased medication use, and decreased quality of life for caregivers and patients.

Despite the fact that animal assisted therapy has been used for many years, some senior healthcare settings still do not accept animals, even though they acknowledge the positive benefits of animal assisted therapy and activities involving therapy pets. Many are concerned regarding the negative effects to human beings such as allergies, infections, biting, scratching, or even fear of the animals involved in therapy [4].

Consequently, robotic pet therapy is seen as a viable substitute for animal therapy. The PARO (short for "personal robot" in the Japanese language) Robotic Pet has been in use in many countries since 2003. The FDA-approved device is designed to look like a baby harp seal, which is a non-familiar animal to most people. As a result, individuals can readily engage with PARO without preconceptions or expectations. Covered in artificial fur, the robotic creature has a hard inner skeleton under which there are dual processors that control software for behavior generation and voice recognition. PARO, as a result, imitates animal behavior, but also responds to light, sound temperature, touch and posture and, over time, due to its artificial intelligence capability, develops its own "character." PARO promotes the therapeutic results of psychological, physiological, and social effort from those who interact with it, lowering stress, improving depression, and reducing anxiety in many cases $[5,6]$. Accurate data was needed regarding the effectiveness of robotic pet therapy. Therefore, the primary purpose of this study was to rigorously assess the effectiveness of PARO robotic pet therapy in treating dementia-related symptoms such as anxiety and depression.

\section{MATERIALS AND METHODS}

A randomized block design with repeated measurements guided the study. The before and after outcome measures included: Rating for Anxiety in Dementia (RAID), Cornell Scale for Depression in Dementia (CSDD), Global Deterioration Scale (GDS), pulse rate, pulse oximetry, galvanic skin response (GSR), and medication utilization. The interventional group received treatment with the PARO robotic pet three times a week for 20 minutes, and the control group received the standard of care, which includes music, physical activity, and mental stimulation in 20-minute segments.
IRB approval and ethics committee approval were obtained. Facility letters of support were also obtained. Education was provided for the facility nurses and staff regarding the purpose of the study and the protocols for data collection. Subjects who met inclusion/exclusion criteria, or their significant family member, were approached and consented. Participation in the study was voluntary, and all residents had the right to refuse. Subjects routinely participated in group programming activities throughout the day. Subject groups were randomly assigned by the toss of a coin to receive either the PARO robotic pet or standardized programming.

Both interventional and control group subjects were volunteers. Each participant was physiciandiagnosed with mild to moderate dementia, utilizing standard Diagnostic and Statistical Manual (DSM) or the National Institute on Aging Alzheimer's Organization criteria, and was 65 years of age or older. Patients with pre-existing psychiatric diagnoses (bipolar, schizophrenia, personality disorder, etc.) and those unable to participate in programming due to physical limitations were excluded from participating in the study.

Consenting subject groups were randomlyassigned based on a coin toss until sample size was obtained. Cohen's $d$ was used for sample size calculation. A sample of 60 was estimated based on an effect size $(0.35)$ with a power of 0.80 , and a $10 \%$ rate of attrition. The participants were equally divided into study and control groups and each group included 5 subgroups.

Experimental group participants were exposed to treatment with the PARO robotic pet once a day for three days a week. Each session lasted 20 minutes and sessions continued for three months. The PI and the trained facility nurses conducted the sessions with the PARO robotic pets in the activity room of the assisted living memory care units. The 20-minute sessions involved seating 6 residents at a round table, placing the PARO robotic pet in the center of the table, and encouraging the residents to interact with the robotic pet by demonstrating interaction. The demographic data and pre-tests of RAID, CSDD, and GDS were administered by the PI and the trained facility nurses prior to the sessions with the PARO robotic pet as well as after three months of exposure. The GSR, pulse oximeter, and pulse rate were recorded for each subject for every session throughout the three-month time period.

The comparison group received what is considered the facilities' standard of care which includes 
physical activity, music, and mental stimulation. Facility nurses conducted these activities in small groups in the activity room on a daily basis. The demographic data, GDS scores, and pre-tests of RAID and CSDD were administered and collected by the PI and the trained facility nurses and staff prior to the standardized program sessions as well as after three months of exposure. The GSR, pulse rate, and pulse oximetry readings were recorded for each subject before and after every session throughout the three-month time period.

In summary, pulse oximetry, pulse rate, and GSR were collected before and after each 20-minute exposure to the robotic pet. The CSDD and the RAID were utilized for staff observations of selected behaviors prior to and after the study. Residents were assessed as to the severity of their dementia with the GDS before and after the study. Staff observation and measurement of pulse oximetry, pulse rate, and GSR, along with the assessment of severity of dementia with the GDS, occurred in the same manner with a control group of residents, who did not receive treatment with the PARO robotic pet.

The RAID Scale is a reliable and valid scale for measuring anxiety in dementia patients. In a previous study, inter-rater reliability and test/retest reliability were moderate, with an overall agreement of over $80 \%$ for individual items. Scores of 11 and above on the scale indicated significant clinical anxiety. The scale correlated significantly with other anxiety scales and with independent ratings [7]. Criterion validity and construct validity were established when the instrument was piloted on 51 inpatients and 32 day-hospital patients who had a DSM diagnosis of dementia.

The sensitivity and specificity of the CSDD has been reported as the $93 \%$ and $97 \%$, respectively. The CSDD was chosen for this study because its validity as a screening tool for depression dementia patients exceeds the Geriatric Depression Scale in progressing dementia [8]. A score $>10$ indicates a probable major depressive episode. A score $>18$ definite major depressive episode.

Interrater reliability for the GDS was found to be high, ranging from 0.87 to 0.97 in various studies [9]. Concurrent validity of the GDS was established by comparing scores of the GDS to scores from the Mini-Mental State Examination and showed high correlation between the two tools. Clinical/biological validity was also demonstrated to be satisfactory by comparing GDS results with results from psychometric tests $(r=0.30-0.60)$, CT scan measures $(r=0.50$ for sulcal enlargement and 0.60 for ventricular dilation), and cerebral blood flow ( $r=0.70-0.80)$ [10].

Pulse rate and pulse oximetry have long been validated as indicators of stress and anxiety. As stress or anxiety decrease, the pulse rate is decreased. Both of these are autonomic responses that fluctuate regardless of cognitive ability. Pulse oximetry readings improve as stress decreases [11]. GSR, or skin conductivity, can also be used as an indication of one's state of arousal [12]. GSR has been observed to continuously change over time and is correlated to the activity of the eccrine sweat glands. Located in the dermis, the eccrine sweat glands regulate body temperature by manufacturing and excreting sweat onto the skin's surface. GSR can be measured through the collection of skin conductance and used as the quantitative indicator of anxiety [13]. The development of bias potentials and polarization were minimized through the use of silver chloride cup electrodes. Velcro fasteners were used to secure the electrodes to the volar surfaces. Retrospective and concurrent data was collected for utilization of pain, depression, sleep and behavior medications during the course of the study.

\section{RESULTS}

A total of 61 patients (23\% males, $77 \%$ females) with an average age of 83.4 years were randomized into the control and treatment groups. Table 1 summarizes the comparison in baseline characteristics and shows no difference between the two groups, thus relating homogeneity. Compared to the control

Table 1

Comparison in baseline characteristics, values presented as mean (std) unless specified. T test used for continuous variables and Chi-square test for categorical variables

\begin{tabular}{lccc}
\hline & Control $(n=26)$ & Treatment $(n=35)$ & $p$ value \\
\hline Age & $83.3(6.0)$ & $83.5(5.8)$ & 0.873 \\
Male, $n(\%)$ & $6(23.1)$ & $8(22.9)$ & 0.984 \\
Race, $n(\%)$ & & & \\
Black & $0(0)$ & $2(5.9)$ & 0.448 \\
Hispanic & $1(3.9)$ & $1(2.9)$ & \\
White & $25(96.1)$ & $31(91.2)$ & \\
RAID & $9.2(5.5)$ & $12(6.3)$ & 0.070 \\
GDS & $5.3(1.0)$ & $5.6(0.8)$ & 0.155 \\
CSDD & $9.4(4.8)$ & $12.5(6.7)$ & 0.054 \\
GSV & $87.5(5.9)$ & $86.0(5.9)$ & 0.352 \\
PulseOX & $90.3(17.7)$ & $94.8(2.1)$ & 0.202 \\
PulseRate & $77.5(9.5)$ & $75.4(11.1)$ & 0.439 \\
Pain med & $6.1(4.9)$ & $6.9(7.1)$ & 0.639 \\
Sleep med & $3.9(3.5)$ & $3.8(3.5)$ & 0.901 \\
Depression med & $5.3(3.6)$ & $5.8(4.6)$ & 0.648 \\
Behavior med & $7.7(9.7)$ & $9.2(9.1)$ & 0.537 \\
\hline
\end{tabular}


Table 2

Comparison in outcome changes after therapies, values presented as mean (stderr). T test was used

\begin{tabular}{lccc}
\hline Difference (post - pre) & Control & Treatment & $p$ value \\
\hline RAID & $0.55(0.2)$ & $2.5(0.6)$ & 0.003 \\
GDS & $-0.06(0.1)$ & $-0.07(0.03)$ & 0.948 \\
CSDD & $0.78(0.4)$ & $2.81(0.4)$ & 0.001 \\
GSV & $0.99(0.2)$ & $5.47(1.3)$ & 0.0005 \\
PulseOx & $0.46(0.1)$ & $1.66(0.08)$ & 0.0001 \\
PulseRate & $0.08(0.2)$ & $-2.97(0.2)$ & 0.0001 \\
Pain med dose & $0.26(0.5)$ & $-2.22(0.7)$ & 0.005 \\
Sleep med dose & $0(0)$ & $0.03(0.6)$ & 0.955 \\
Depression med dose & $0(0)$ & $-0.68(0.4)$ & 0.083 \\
Behavior med dose & $-0.09(0.09)$ & $-2.09(0.54)$ & 0.0009 \\
\hline
\end{tabular}

group, pulse oximetry and GSV were increased, while RAID, CSDD, pulse rate, pain medication, and behavior medication were significantly decreased in the treatment group. Table 2 displays the average changes in outcome measures in response to the therapy. No differences in GDS staging or sleep medication and depression medication utilization were noted between the two groups. Table 3 contains the $p$ values from multivariable regression representing the change in each outcome after the therapy. After adjusting for demographic variables, the group status showed a significant effect on all outcomes except GDS, sleep medication and behavior medication. Table 4 represents a multivariable regression, using a mixed model, depicting GSV, pulse oximetry, and pulse rate, which were repeatedly measured throughout the study. After adjusting for demographics and time variable, the group status showed a significant effect on GSV, pulse oximetry, and pulse rate. The changes in GSV, pulse oximetry, and pulse rate over time were plotted for both groups in Fig. 1. The difference between the two groups was seen consistently throughout the study for pulse oximetry and pulse rate, while changes in GSV showed no difference between the two groups for several weeks.

\section{DISCUSSION}

Using the data from the three-month study, researchers found that intervention with the PARO
Table 4

$P$ values from multivariable regression analysis - Mixed Model

\begin{tabular}{lccc}
\hline $\begin{array}{l}\text { Independent } \\
\text { Variable }\end{array}$ & \multicolumn{3}{c}{$\begin{array}{l}\text { Dependent variables as difference } \\
\text { between post- and pre- therapies }\end{array}$} \\
\cline { 2 - 4 } & GSV & PulseOx & PulseRate \\
\hline Age & 0.568 & 0.873 & 0.384 \\
Gender & 0.214 & 0.616 & 0.812 \\
Group & 0.008 & 0.0001 & 0.0001 \\
Week & 0.310 & 0.641 & 0.072 \\
Day & 0.817 & 0.209 & 0.564 \\
\hline
\end{tabular}

robotic pet seal provided a viable alternative for controlling symptoms of anxiety and depression in elderly patients with dementia, often in lieu of pharmacological modalities. Oxygen saturation, pulse rate, GSV, RAID, and CSDD and medication use were all positively impacted in patients participating in the interventional group, indicating improvement in symptom control.

This study significantly contributes to the body of knowledge regarding robotic biofeedback devices in the treatment of dementia. The study utilized a robust sample, measured a variety of outcomes, and used a randomized design. Previous studies lacked rigor with regard to sample size, design, and controlling for extraneous measures. Caution should be utilized in generalizing results beyond the sample in this study, however, due to the unique population of subjects.

Implications for practice for providers working with this population include: 1) the average individual in the senior living environment consumes on average 16 to 28 medications per day at an average cost of 1200 to 1500 U.S. dollars per month [14]; and, 2) intervention with the PARO robotic pet three times weekly for 20 minutes significantly reduced the need for these medications. As the literature suggests, the use of benzodiazepines in the elderly population result in falls, sedation, and physical dependence [15]. Additionally, providers may use antipsychotics off-label to treat negative behaviors in individuals with dementia; these medications can cause or worsen heart arrhythmias in the older adult and worsen other chronic conditions such as renal impairment, GI distress, and liver impairment [16]. Use of pain medications in the treatment group was

Table 3

$P$ values from multivariable regression analysis - General Linear Model (GLM)

\begin{tabular}{lccccccc}
\hline Independent Variable & \multicolumn{7}{c}{ Dependent variables as difference between post- and pre- therapies } \\
\cline { 2 - 8 } & RAID & GDS & CSDD & PainMed & SleepMed & DepressionMed & BehaveMed \\
\hline Age & 0.738 & 0.699 & 0.563 & 0.847 & 0.549 & 0.540 & 0.678 \\
Gender & 0.914 & 0.478 & 0.401 & 0.743 & 0.321 & 0.356 & 0.689 \\
Group & 0.005 & 0.912 & 0.001 & 0.009 & 0.910 & 0.138 & 0.003 \\
\hline
\end{tabular}


GSV: Post-Pre

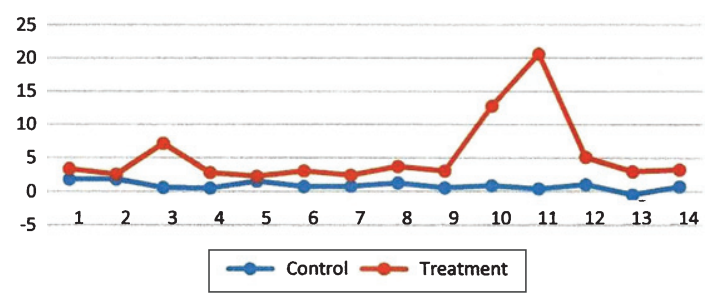

PulseOx: Post-Pre

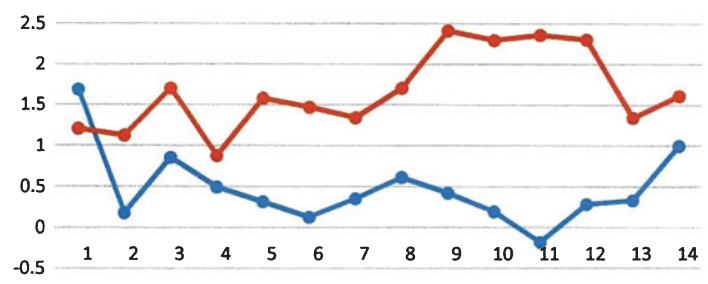

$-0.5 \sim$ Control $\backsim$ Treatment

Pulserate: Post-Pre

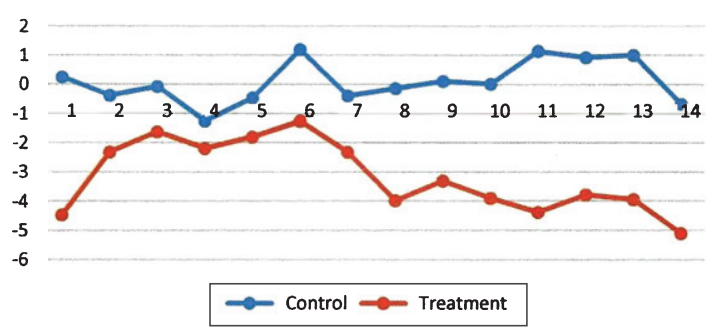

Fig. 1. Profiling of changes in biometric measures over time.

significantly decreased as well, which may lead to further utilization of the PARO. Other applications for the PARO, in addition to reducing stress (as measured by GSV), may include improved oxygenation and improved cardiac status. The treatment group improved and maintained improvements in GSV, pulse oximetry, and pulse-rate over time (Fig. 1).

Whereas depression scores improved with the treatment groups, providers were reluctant to discontinue or reduce the amount of antidepressant medication. Further research is needed regarding provider awareness of evidence-based results of nontraditional methods of treating depression such as biofeedback therapy with robotic pets and provider willingness to discontinue or decrease medication use when depression scores improve with such therapy.

Significant improvements in observed pain and decreased pain medication use were noted in the interventional group. Recent literature advises an observed overlap between pain and psychiatric disorders is common because some neurotransmitters, such as serotonin and norepinephrine (typically lower in individuals with dementia), are involved, albeit in different brain regions, in pain and sensory processing, as well as in modulating mood [17]. Thus, it is likely that treatment with the PARO, which decreases stress and anxiety, will also be effective in controlling or assisting in the relief of chronic pain.

Future research could examine the impact of biofeedback therapy with robotic pets in acute care settings with various patient populations. Other physiological measures could be considered to measure the impact of interventions with the PARO robotic pet seal, especially varying exposure of the treatment regimen.

\section{ACKNOWLEDGMENTS}

The authors wish to acknowledge the Baylor Deerbrook Charitable Trust that funded the study. Appreciation is also expressed to the leadership, nurses, staff and families at Signature Senior Living of Dallas, Texas and Legend Senior Living of Wichita, Kansas who took part in the study.

Authors' disclosures available online (http://j-alz. com/manuscript-disclosures/16-0703r1).

\section{REFERENCES}

[1] Alzheimer's Disease International (2013) Policy Brief for G8 Heads of Government. The Global Impact of Dementia 2013-2050, Alzheimer's Disease International, London.

[2] Byers A, Yaffe K (2011) Depression and risk of developing dementia. Nat Rev Neurol 7, 323-331.

[3] Yu R, Hui E, Lee J, Poon D, Ng A, Sit K, Ip K, Yeung F, Wong M, Shibata T, Woo J (2015) Use of a therapeutic, socially assistive pet robot (PARO) in improving mood and stimulating social interaction and communication for people with dementia: Study protocol for a randomized controlled trial. JMIR Res Protoc 4, e45.

[4] Velde B, Joseph C, Fisher G (2005) Resident and therapist views of animal-assisted therapy: Implications for occupational therapy practice. Aust Occup Ther J 52, 43-50.

[5] Broekens J, Heerink M, Rosendal H (2009) Assistive social robots in elderly care: A review. Gerontechnology 8, 94-103.

[6] Bemelmans R, Gelderblom G, Jonker P, de Witte L (2012) Socially assistive robots in elderly care: A systematic review into effects and effectiveness. J Am Med Dir Assoc 13, 114120.

[7] Shankar K, Walker M, Frost D, Orrell M (1999) The development of a valid and reliable scale for rating anxiety in dementia (RAID). Aging Ment Health 3, 39-49.

[8] Kørner A, Lauritzen L, Abelskov K, Gulmann N, Marie Brodersen A, Wedervang-Jensen T, Marie Kjeldgaard K (2006) The Geriatric Depression Scale and the Cornell Scale for Depression in Dementia. A validity study. Nord J Psychiatry 60, 360-364. 
[9] Herndon R (2006) Handbook of Neurologic Rating Scales (2nd ed.), Demos Medical Publishing, New York.

[10] Eisdorfer C, Cohen D, Paveza G, Ashford J, Luchins D, Gorelick P, Hirschman R, Freels S, Levy P, Semla T, Shaw H (1992) An empirical evaluation of the Global Deterioration Scale for staging Alzheimer's disease. Am J Psychiatry 149, 190-194.

[11] National Center for Complementary and Alternative Medicine (US). Relaxation Techniques for Health: An Introduction [Internet]. Health Information. NCCIH Pub. No: D461, http://nccam.nih.gov/health/stress/relaxation. htm\#status, Last updated May 2016. Accessed on June 1, 2016.

[12] Boucsein W (1992) Electrodermal Activity, Plenum University Press, New York.

[13] Stern R, Ray W, Quigley K (2001) Psychophysiological Recording, 2nd ed, Oxford University Press, New York.
[14] IMS Health Incorporated; Special Data Request, 2016. Calculations based on 2014 population estimates from the U.S. Census Bureau, http://kff.org/other/state-indicator/ retail-rx-drugs-by-age/, Last updated December 2016. Accessed on June 1, 2016.

[15] Sithamparanathan K, Sadera A, Leung L (2012) Adverse effects of benzodiazepine use in elderly people: A metaanalysis. Asian J Gerontol Geriatr 7, 107-111.

[16] Gareri P, Segura-García C, Graziella V, Manfredi L, Bruni A, Ciambrone P, Cerminara G, De Sarro G, De Fazio P (2014) Use of atypical antipsychotics in the elderly: A clinical review. Clin Interv Aging 9, 1363-1373.

[17] Barry D, Pilver C, Hoff R, Potenza M (2013) Pain interference and incident mood, anxiety, and substance-use disorders: Findings from a representative sample of men and women in the general population. J Psychiatr Res 47, $1658-1664$. 